\title{
A Review on Implementation of Generating a Path Backup for Handling Faults in MANETs
}

\author{
Neelam Rani ${ }^{1}$, Ms. Amrita Chaudhary ${ }^{2}$ \\ M.Tech Scholar, Computer Science \& Engineering Dept, Galaxy Global Group of Institutes, Kurukshatra ${ }^{1}$ \\ Asst. Professor, Computer Science \& Engineering Dept, Galaxy Global Group of Institutes, Kurukshatra ${ }^{2}$
}

\begin{abstract}
Mobile Ad-Hoc Network (MANET) is a collection of wireless mobile hosts forming a temporary network without the aid of any stand-alone infrastructure or centralized administration. Due to the mobility of the nodes in the network, these nodes are self-organizing and self-configuring. Not only they act as hosts, but also they function as routers. This paper presents a review on generating a backup path after handling some faults in primary path. Therefore, an efficient route between any two nodes with minimum routing overhead and bandwidth consumption should be established. The design of these routing protocols is challenging due to the mobility and the dynamic nature of the mobile ad-hoc networks. A performance comparison will be conducted to analyse the performance of system. This will use MATLAB tool for simulation.
\end{abstract}

Keywords: MANET, AODV, Shortest Path, Reactive Routing Protocol etc.

\section{INTRODUCTION}

A Computer networks were originally developed to operate by connecting computers together with wires and transmitting data over these wires. Network sizes and occurrences increased creating a requirement for inter-network communication. This led to the development of the Internet and its suite of protocols. The use of the Internet and its applications became ubiquitous. A need for providing network access to entities while not physically attached to the wired network arose. To enable this wireless networking was developed, providing devices with methods to connect to a wired network using radio wave technologies through wireless access points. Simultaneously, telephone networks were undergoing a similar transformation.

Cellular network technologies were developed to allow mobile phones to connect via base stations and communicate in a circuit switched environment. The area of mobile ad-hoc networking deals with devices equipped to perform wireless communication and networking, but without any existing infrastructure such as base stations or access points. Wireless devices form a network as they become aware of each other's presence. They communicate directly with devices inside their radio range in a peer-to-peer nature and to communicate with a device outside their range, they can use an intermediate device or devices within their radio range to relay or forward communications to the device outside their range [1].

An ad-hoc network is self-organizing and adaptive. Networks are formed on-the-fly; devices can leave and join the network during its lifetime, devices can be mobile within the network, the network as a whole may be mobile and the network can be deformed on the-fly. All this needs to be done without any system administration and without the requirement for any permanent devices within the network. The mobile ad-hoc network is a collection of wireless mobile nodes forming a temporary network without centralized administration. Mobile Ad-hoc networks are self-organizing and self-configuring multi-hop wireless networks where, the structure of the network changes dynamically, due to the mobility of the nodes. Nodes in these networks utilize the same random access wireless channel and multi-hop forwarding. The nodes in the network not only act as hosts but also as routers that transfers data to/from other nodes in network.

Mobile Ad Hoc Networks (MANETS) are wireless mobile nodes that cooperatively form a network without infrastructure. In other words, ad hoc networking allows devices to create a network on demand without prior coordination or configuration. Thus, nodes within a MANET are involved in routing and forwarding information between neighbours, because there is no coordination or configuration prior to setup of MANET. MANET is selfconfiguring networks of mobile nodes without the presence of static infrastructure. They can also be heterogeneous, which means that all nodes don't have the same capacity in term of resources (power consumption, storage, computation, etc.). Due to its great features, MANET attracts different real world applications areas where the networks topology changes very quickly. A good example is given by military battlefield networks. In that case, mobile devices have different communications capability such as radio range, battery life, data transmission rate, etc. 
Vol. 6, Issue 4, April 2017

The paper is organized as follows. In section II, we discuss related work with the motion estimation and compensation. In Section III, It describes introduction of video coding. In Section IV, it describes the introduction of motion estimation. Section V defines the proposed technique related to system. Finally, conclusion is given in Section VI.

\section{RELATED WORK}

Radwan S. Abujassar (2015)[1] evaluated the impact of backup path where the nodes move continuously and change their speed (from walking speed to bike speed). The nodes are expecting to receive the demand traffic even when they are changing their positions. The backup route was computed through routing table to alleviate the impact of this on the network by finding an alternative path ready to use to pass the traffic when the primary one fails.

Cherry Ye Aung et. Al. [2] reviewed mobility models proposed for MANETs. They also discussed the potential of using or adapting these mobility models for other emerging network types. They have reviewed some of the most representative group mobility models proposed for MANET research. They classified the models into four categories based on the movement of the group members. They concisely described how the group and group member's move in each model based on their classification.

V. Hema, M. Mohanapriya (2014) [3] proposed a new Rebroadcast Technique for Reducing Routing Overhead in Mobile Ad Hoc Networks (MANETs). The neighbour coverage knowledge comprises of coverage ratio and the connectivity factor. This technique dynamically calculates the rebroadcast delay, which finds the forwarding order and also effectively find the neighbour coverage knowledge. The result of the simulation showed that the new mechanism produces less rebroadcast than the existing protocol..

Gulfishan Firdose Ahmeda et. Al. (2012)[4] examined routing protocol for mobile ad hoc networks the Destination Sequenced Distance Vector (DSDV) and On packet delivery fraction and average delay while varying number of sources and pause time. In this paper they considered the new approach an Improved-DSDV Protocol is proposed for Ad Hoc networks. Improved -DSDV overcomes the problem of stale routes, and thereby improves the performance of regular DSDV.

Ma Xiang (2012) [5] proposed a QOS gateway discovery protocol which uses the time delay and stable route to the gateway selection conditions. And according to the gateway discovery protocol, it also proposed a fast handover scheme which can decrease the handover time and improve the handover efficiency. This paper presented a QOS gateway discovery protocol which uses the time delay and stable route to the gateway and the gateway selection parameters and according to the gateway discovery protocol, it also proposes a fast handover scheme which can decrease the handover time and improve the handover efficiency.

Laxmi Shrivastava et. Al. [6] presented a detailed performance comparison of important routing protocols for mobile ad-hoc networks. AODV and DSR are reactive protocol while DSDV is a proactive protocol. Both AODV and DSR use reactive approach to route discovery, but with different mechanism. DSR uses source routing and route cache and does not depend on their timer base activity. On other hand AODV uses routing tables, one route per destination, sequence number to maintain route. The general observation from simulation is that DSR has performed well compared to AODV and DSDV in the situation of heavy traffic load.

S. Mohapatraa et. Al. (2011) [7] they evaluated the four performance measures i.e. control overhead, PDR, end-to-end delay and throughput with different number of nodes, different speed (pause time) of nodes and different size of network. They concluded that DSR protocol is the best in terms of average PDR. For high mobility condition of nodes DSR gives better packed delivery ratio than other protocols making it suitable for highly mobile random networks. they consider $600 \times 600 \mathrm{sqm}$ size network to evaluate the network load anlaysis and mobility analysis. If the network size is more than 600x600sqm.and if PDR and throughput are the prime criteria, the OLSR protocol is the better solution for high mobility condition.

Dr. Chandra Shekar Reddy Putta et. Al. [8] they have evaluated the performance of reactive (i.e. DSR and AODV) and proactive (i.e. OLSR) routing protocols in 802.11 ad-hoc network environment. We have noticed the proactive protocol (OLSR) offers better performances for CBR sources (eg. voice services) given that it guaranties lowest delay. However it consumes much more bandwidth. Periodically, OLSR protocol sends routing packets to discover and to maintain routes to all destinations. That's why the number of delivered packets decreases when the traffic load (number of connections increases. For 10 connections, the packet delivery ratio is about $53 \%$. The reactive routing protocols are more adapted for data services (file transfer). They guarantee a packet delivery ratio of $80 \%$ for 60 connections.

Emmanuel Baccelli et. Al. (2010)[9] they have overviewed the key challenge with routing on multi-hop wireless networks with OSPF: drastic control signalling reduction while keeping track of a topology that changes much more often compared to "usual" OSPF topology. A distinct category of solutions to this problem was identified as being different configurations of the same concept, derived from multi-point relay (MPR) techniques.

Ying-Hong Wang et. Al. (2006)[10] a new on demand routing protocol for MANETs, DBR2P, is proposed. It could provide a backup node mechanism for quick reconnection when a link fails and set up many routes to reach a destination in a given period. Even when a link fails, these routes, almost more than one, from the source node to the 
destination node can be analysed to obtain backup routes to support reconnection.DBR2P is proposed to focus attention on the intrinsic properties of MANETs and the results of the simulation experiment show that DBR2P has good performance.

\section{MANET ROUTING PROTOCOLS}

Routing is the most fundamental aspect for multi-hop MANETs. Unlike the Internet and infrastructure-based wireless networks, MANETs are characterized by the lack of a dedicated routing infrastructure. MANET nodes depend on each other to forward traffic. This requires nodes to forward traffic on behalf of other nodes, which opens the door for selfish behavior. Selfish behavior can significantly degrade performance of routing protocols that assume honest nodes [2]. In addition, Manet's topology undergoes continuous reconfiguration due to the dynamic nature of wireless links and node mobility. The research community has responded to these challenges by proposing many routing protocols. Most of these protocols can be classified as follows: (1) proactive routing, (2) reactive routing and (3) geographical routing.

\section{A. Proactive Routing}

In proactive routing protocols nodes periodically exchange routing control messages and establish routes to all other nodes. Proactive routing can be implemented based on either: (1) link-state or (2) distance-vector.

\section{Link-State Routing}

In link-state routing nodes periodically use "HELLO" and "TOPOLOGY CONTROL(TC)" messages to discover and disseminate learned information about node connectivity. This information describes the state of links connecting different nodes. Each node, S, periodically broadcasts a "HELLO" message. Every one hop neighbor of S responds thus informing $\mathrm{S}$ they are neighbors. $\mathrm{S}$ then generates and broadcasts a TC message, which is flooded throughout the network. TC messages inform other nodes about the connectivity between $\mathrm{S}$ and its immediate neighbors. They allow each node to construct a network map and compute the next hop, or the entire path, to all destinations. This can be easily achieved using the Dijkstra shortest path algorithm.

\section{Distance-Vector}

In distance-vector, nodes periodically construct and broadcast routing tables containing the reachable destinations and the number of hops to reach them, or some other cost metric. Nodes continuously update their own routing tables based on tables they receive from their neighbours. A distributed variant of Bellman-Ford algorithm is at the core of most distance-vector routing protocols. The main disadvantages of distributed Bellman-Ford algorithm are: (1) it does not scale well, (2) changes in network topology are not reflected quickly because updates are spread node-by-node, (3) the count to infinity problem.

\section{B. Reactive Routing}

In bandwidth and power-limited environments, it is desirable to minimize routing control traffic when there is no data to be routed. Proactive routing is not particularly suitable for such settings. This is the main motivation behind reactive routing, also called on-demand routing. Such protocols do not maintain routes, but build them on-demand when communication to a certain destination is required. For example, the ad-hoc on-demand distance-vector (AODV) protocol is a reactive adaptation of distance-vector.

\section{Position-Based Routing}

In position-based routing, also called geographic routing or geo-routing, nodes rely on position information to forward traffic from a source to a destination. The main idea is that the source sends a message to a destination's geographic location, instead of its network address. This requires each node to determine its own location, and the source to be able to deduce that of the destination. A message can be routed based on the geographic destination, without knowledge of the network topology, or prior route discovery.

\section{DESCRIPTION OF PROTOCOLS USED}

\section{A. Ad hoc On Demand Distance Vector Routing (AODV)}

Reactive protocol is identified as On-demand protocols because it creates routes only when these routes are needed. The need is initiated by the source, as the name suggests. When a sender node requires a route to a destination, it initiates a route discovery process within the network. This process is completed once a route is found or all possible route permutations have been examined. Ad hoc On-Demand Distance Vector (AODV) routing is a routing protocol for mobile ad hoc networks and other wireless ad-hoc networks. It is jointly developed in Nokia Research Centre of University of California, Santa Barbara and University of Cincinnati by C. Perkins and S. Das. It is an on-demand and distance-vector routing protocol, meaning that a route is established by AODV from a destination only on demand. 
AODV is capable of both unicast and multicast routing [17]. It keeps these routes as long as they are desirable by the sources. Additionally, AODV creates trees which connect multicast group members. The trees are composed of the group members and the nodes needed to connect the members. The sequence numbers are used by AODV to ensure the freshness of routes. It is loop-free, self-starting, and scales to large numbers of mobile nodes. AODV defines three types of control messages for route maintenance:

RREQ-A route request message is transmitted by a node requiring a route to a node. As an optimization AODV uses an expanding ring technique when flooding these messages. Every RREQ carries a time to live (TTL) value that states for how many hops this message should be forwarded. This value is set to a predefined value at the first transmission and increased at retransmissions. Retransmissions occur if no replies are received.

RREP- A route reply message is uni-casted back to the originator of a RREQ if the receiver is either the node using the requested address, or it has a valid route to the requested address. The reason one can unicast the message back, is that every route forwarding a RREQ caches a route back to the originator.

RERR-Nodes monitor the link status of next hops in active routes. When a link breakage in an active route is detected, a RERR message is used to notify other nodes of the loss of the link. In order to enable this reporting mechanism, each node keeps a - precursor list", containing the IP address for each its neighbors that are likely to use it as a next hop towards each destination.

\section{B. Dynamic Source Routing (DSR)}

Dynamic Source Routing (DSR) is a routing protocol for wireless mesh networks. It is similar to AODV in that it establishes a route on-demand when a transmitting mobile node requests one. However, it uses source routing instead of relying on the routing table at each intermediate device. Dynamic source routing protocol (DSR) is an ondemand, source routing protocol [27], whereby all the routing information is maintained (continually updated) at mobile nodes.

DSR allows the network to be completely self-organizing and self-configuring, without the need for any existing network infrastructure or administration. The protocol is composed of the two main mechanisms of "Route Discovery" and "Route Maintenance", which work together to allow nodes to discover and maintain routes to arbitrary destinations in the ad hoc network. An optimum path for a communication between a source node and target node is determined by Route Discovery process. Route Maintenance ensures that the communication path remains optimum and loop-free according the change in network conditions, even if this requires altering the route during a transmission. Route Reply would only be generated if the message has reached the projected destination node (route record which is firstly contained in Route Request would be inserted into the Route Reply).

To return the Route Reply, the destination node must have a route to the source node. If the route is in the route cache of target node, the route would be used. Otherwise, the node will reverse the route based on the route record in the Route Reply message header (symmetric links). In the event of fatal transmission, the Route Maintenance Phase is initiated whereby the Route Error packets are generated at a node. The incorrect hop will be detached from the node's route cache; all routes containing the hop are reduced at that point. Again, the Route Discovery Phase is initiated to determine the most viable route.

\section{PROPOSED WORK}

MANET performance is sensitive to mobility, scalability and traffic load, so to examine the different protocol performance while the amount of traffic and speed of nodes varies even plays a crucial role in efficient traffic routing. The reactive protocols determine a route only when necessary. The source node is the one in charge of the route discovery. As a main advantage, the routing overhead is small since the routes are determined only on demand. As a main disadvantage the route discovery introduces a big delay. Due to this, this thesis proposes a routing protocol for MANETs with the objective that each node works using the most suitable features. To achieve that, every node checks periodically its speed and its traffic.

\section{CONCLUSION}

There occurs a problem of broken links due to the lack of energy which cause disorder in network system. Such problem occurs due to the unawareness of energy of mobile neighbour nodes. In this study, it proposes an efficient algorithm for MANETs, which will maximize the network lifetime. It will be used to determine the local link connectivity information for monitoring the link status between nodes along with the incorporation of Dynamic ON Demand Routing Protocol to reduce the energy consumption of mobile nodes to certain extent. These protocols will use shortest path as a main metric to establish routing between source and destination. In this, it will provide a review on comparison of proposed protocol with AODV. 


\section{REFFERENCES}

[1] R. Li Radwan S. Abujassar, "Mitigation fault of node mobility for the MANET networks by constructing a backup path with loop free: enhance the recovery mechanism for pro-active MANET protocol”, in the proceedings of Springer Science+Business Media ,2015

[2] Cherry Ye Aung,Boon Chong Seet, Mingyang Zhang, "A Review of Group Mobility Models for Mobile Ad Hoc", in the proceedings of Springer Science+Business Media 2015.

[3] V.Hema, M.Mohanapriya, "Routing Overhead Reduction in MANETs", in the proceedings of International Journal Of Scientific \& Engineering Research, Volume 5, Issue 3,pp.27-32, March-2014.

[4] Gulfishan Firdose Ahmeda, Raju Barskar and Nepal Barskar, "An Improved DSDV Routing Protocol For Wireless Ad hoc", in the proceedings of International Conference on Communication, Computing \& Security (ICCCS),pp.822-831,2012.

[5] Ma Xiang, "Research of Ad Hoc Networks Access Algorithm', in the proceedings of International Conference on Solid State Devices and Materials Science Elsevier, pp. 2257 - 2262, 2012.

[6] Laxmi Shrivastava, Sarita S.Bhadauria, G.S.Tomar,'Performance Evaluation of Routing Protocols in MANET with different traffic loads', in the proceedings of IEEE,(International Conference on Communication Systems and Network Technologies), pp.13-16,2011.

[7] Mohapatraa,P.Kanungo,"Performance analysis of AODV, DSR, OLSR and DSDV Routing Protocols using NS2 Simulator'” in the proceedings of International Conference on Communication Technology and System Design Published by Elsevier Ltd. Pp. 69-76, 2011.

[8] Dr. Chandra Shekhar Reddy Putta,Dr. K.Bhanu Prasad ,Dilli Ravilla,"Performance of Ad hoc Network RoutingProtocols', in the preceedings of IEEE (Int'l Conf. on Computer \& Communication Technology,pp.372-376,2010.

[9] Emmanuel Baccelli, Juan Antonio Cordero and Philippe Jacquet, " OSPF OVER MULTI-HOP AD HOC WIRELESS COMMUNICATIONS', in the proceedings of International Journal of Computer Networks \& Communications (IJCNC) Vol.2, No.5, pp.37$56,2010 . S$

[10] Ying-Hong Wang, Chih-Feng Chao, "Dynamic backup routes routing protocol for mobilead hoc networks", in the proceedings of Information Sciences, pp.161-185,2006.[11]Fei Dai and Jie Wu, “ Proactive Route Maintenance in Wireless Ad Hoc Networks”, in proceedings of IEEE communications, 2005. 